\title{
Extended rational sinh-cosh and sin-cos methods to derive solutions to the coupled Higgs system
}

\author{
Hadi Rezazadeh ${ }^{1}$, Alper Korkmaz ${ }^{2, *}$, Mostafa Eslami $^{3}$, Jian-Guo Liu ${ }^{4}$ \\ ${ }^{I}$ Faculty of Engineering Technology,Amol University of Special Modern Technologies, Amol, Iran \\ ${ }^{2}$ Department of Mathematics, CAKU, Çankırı, Turkey \\ ${ }^{3}$ Department of Mathematics, Faculty of Mathematical Sciences, University of Mazandaran, Babolsar, Iran \\ ${ }^{4}$ College of Computer, Jiangxi University of Traditional Chinese Medicine, Jiang Xi 330004, China
}

\begin{abstract}
By using the new recently proposed extended rational methods, we set some solitary and multi wave solutions of the coupled Higgs system in the present study. Some new families of hyperbolic and trigonometric solutions of the coupled Higgs equations are successfully obtained. Some solutions are real as some are complex valued functions. Particular forms of some solutions derived by choice of some parameters are demonstrated in three dimensional spaces. The complex valued solutions are also depicted by plotting both real and complex component.
\end{abstract}

Keywords: Coupled Higgs equations, new extended rational methods, traveling wave solution, complex solution, multi wave solution.

\section{Introduction}

The coupled Higgs (field) equation was appeared in early 80 s in the form

$$
\left\{\begin{array}{l}
u_{t t}-u_{x x}-c u+r|u|^{2} u-2 u v=0, \\
v_{t t}+v_{x x}-r\left(|u|^{2}\right)_{x x}=0,
\end{array}\right.
$$

for the choice $c>0, r>0$ [1]. Its name is changed to the coupled nonlinear Klein-Gordon equation for both $c$ and $r$ are negative. The coupled equation given above has $\mathrm{N}$-soliton solutions that can be determined by Hirota's bilinear approach [1]. This coupled Higgs equation defines interacting a conserved scalar complex field of nucleons $u(x, t)$ and neutral scalar real field of mesons $v(x, t)[2,3]$. Both Lie symmetry and $\frac{G^{\prime}}{G}$-expansion approaches were implemented to the coupled Giggs equation to derive its solutions in terms of elliptic functions of Jacobi, hyperbolic, trigonometric and rational functions [4]. Periodic solutions in in infinite numbers and existence of new wave solutions in solitary forms were discussed by the dynamical system bifurcation theory

${ }^{*}$ Corresponding author: akorkmaz@ karatekin.edu.tr (Alper Korkmaz)

E-mail address 
[5]. The conditions to guarantee the existence of these solutions were also summarized in the same study. Various solutions in hyperbolic or trigonometric function forms were determined by implementation of exponential function technique [6]. Jacobi type periodic, hyperbolic and trigonometric solutions in wave forms were set by an algebraic method [7].

In the present study, we focus on the coupled Higgs system [8-10]

$$
\left\{\begin{array}{l}
u_{t t}-u_{x x}+|u|^{2} u-2 u v=0 \\
v_{t t}+v_{x x}-\left(|u|^{2}\right)_{x x}=0
\end{array}\right.
$$

A homoclinic solution to the Higg system (1) was presented in [8] by assistance of Hirota's bilinear technique. (G'/G)-expansion and He's semi inverse methods were also used to set trigonometric and hyperbolic function solutions covering the complex ones [9]. Solutions determined by $\exp (-\varphi(\varepsilon))$-expansion method in forms of rational, trigonometric and hyperbolic functions were summarized in [10].

In the last several years, various smart and effective methods have been derived to solve nonlinear PDEs, systems and even fractional PDEs. Functional variable method [11,12], first integral approach [13-14], a more general form of the method of Bernoulli equation (Kudryashov's method) [15-16], extended hyperbolic tangent fucntion method [17], a finite expansion method based on Sine-Gordon equation [18], the approach of sub equation [19], generalized form of the projective Riccati approach [20], $\exp (-\varphi(\varepsilon))$-expansion method [21], the predicted solution method of hyperbolic function [22,23], the unified method [24] are only some of them. Extended rational methods [25] are also effective techniques to determine solutions to nonlinear PDEs.

In the present study, we implement some extended rational methods in hyperbolic and trigonometric forms to derive the solution to the Higgs system (1). Both procedures start by choice of the predicted solutions with various parameters. The aim in the techniques are to determine the relations among these parameters by using algebraic approaches. The brief description of the method and the solutions are presented in details below.

\section{Description of themethods}

In this section we describe the first step of the new extended rational methods for finding exact solutions of partial differential equations (PDEs)

$$
F\left(u, \frac{\partial u}{\partial t}, \frac{\partial u}{\partial x}, \frac{\partial u^{2}}{\partial x}, \frac{\partial^{2} u}{\partial x^{2}}, \ldots\right)=0
$$

where $u=u(x, t)$ is an unknown function and $F$ is a polynomial in $u$ and its various partial derivatives

Suppose that 


$$
u(x, y, t)=u(\xi), \quad \xi=x+c t,
$$

where $c$ is constant.

Then, by using (3),Eq. (2) can be turned into the following ordinary differential equation (ODE) with respect to the variable $\xi$

$$
G\left(u, u^{\prime}, u^{\prime \prime}, \ldots\right)=0
$$

In two next subsection, we express the main step for finding the exact solutions of Eq.(2) by using the extended rational sinh-cosh method and the extended rational sin-cos method.

\subsection{Extended rational sinh-cosh method}

According to extended rational sinh-cosh method, which was introduced by Darvishi et. al., [25], we assume that the exact solution can be expressed in the following forms

$$
\begin{aligned}
& u(\xi)=\frac{a_{0} \sinh (\mu \xi)}{a_{2}+a_{1} \cosh (\mu \xi)}, \quad \cosh (\mu \xi) \neq-\frac{a_{2}}{a_{1}}, \\
& u(\xi)=\frac{a_{0} \cosh (\mu \xi)}{a_{2}+a_{1} \sinh (\mu \xi)}, \quad \sinh (\mu \xi) \neq-\frac{a_{2}}{a_{1}},
\end{aligned}
$$

where $a_{0}, a_{1}$ and $a_{2}$ are parameters to be found in terms of the other parameters. The nonzero constant $\mu$ is the wave number. The derivatives of the predicted solutions are

$$
\begin{aligned}
& u^{\prime}(\xi)=\frac{a_{0} \mu\left[\cosh (\mu \xi) a_{2}+a_{1}\right]}{\left[a_{2}+a_{1} \sinh (\mu \xi)\right]^{2}}, \\
& u^{\prime \prime}(\xi)=-\frac{a_{0} \mu^{2} \sinh (\mu \xi)\left[2 a_{1}^{2}+a_{1} \cosh (\mu \xi) a_{2}-a_{2}^{2}\right]}{\left[a_{2}+a_{1} \cosh (\mu \xi)\right]^{3}},
\end{aligned}
$$

in the first form and

$$
\begin{aligned}
u^{\prime}(\xi) & =\frac{a_{0} \mu\left[\sinh (\mu \xi) a_{2}-a_{1}\right]}{\left[a_{2}+a_{1} \sinh (\mu \xi)\right]^{2}}, \\
u^{\prime \prime}(\xi) & =\frac{a_{0} \mu^{2} \cosh (\mu \xi)\left[2 a_{1}^{2}-a_{1} \sinh (\mu \xi) a_{2}+a_{2}^{2}\right]}{\left[a_{2}+a_{1} \sinh (\mu \xi)\right]^{3}} .
\end{aligned}
$$

in the second form. 
We substitute Eqs. (7)or(8)into the reduced form of the governing equation obtained above in Eq. (4). Then, equating the coefficients of each term having the same power of $\cosh ^{m}(\mu \xi)$ or $\sinh ^{m}(\mu \xi)$ to zero, an algebraic system of equations including all the parameters used in the predicted solution, governing equation and the wave transform is solved for $a_{0}, a_{1}, a_{2}, c$ and $\mu$. Determining $a_{0}, a_{1}, a_{2}, c$ and $\mu$ in terms of other parameters and substitution of them into Eq. (5) and (6),one gets solutions for Eq.(2) in rational sinh-cosh forms.

\subsection{Extended rational sin-cos method}

According to extended rational sin-cos method, we assume that the exact solution can be expressed in the following forms

$$
\begin{aligned}
& u(\xi)=\frac{a_{0} \sin (\mu \xi)}{a_{2}+a_{1} \cos (\mu \xi)}, \quad \cos (\mu \xi) \neq-\frac{a_{2}}{a_{1}}, \\
& u(\xi)=\frac{a_{0} \cos (\mu \xi)}{a_{2}+a_{1} \sin (\mu \xi)}, \quad \sin (\mu \xi) \neq-\frac{a_{2}}{a_{1}},
\end{aligned}
$$

where $a_{0}, a_{1}$ and $a_{2}$ are parameters to be found in terms of the other parameters. The nonzero constant $\mu$ is the wave number. The derivatives of the predicted solutions are

$$
\begin{aligned}
& u^{\prime}(\xi)=\frac{a_{0} \mu\left[\cos (\mu \xi) a_{2}+a_{1}\right]}{\left[a_{2}+a_{1} \sin (\mu \xi)\right]^{2}}, \\
& u^{\prime \prime}(\xi)=\frac{a_{0} \mu^{2} \sin (\mu \xi)\left[2 a_{1}^{2}+a_{1} \cos (\mu \xi) a_{2}-a_{2}^{2}\right]}{\left[a_{2}+a_{1} \cos (\mu \xi)\right]^{3}},
\end{aligned}
$$

in the first form and

$$
\begin{aligned}
& u^{\prime}(\xi)=-\frac{a_{0} \mu\left[\sin (\mu \xi) a_{2}+a_{1}\right]}{\left[a_{2}+a_{1} \sin (\mu \xi)\right]^{2}}, \\
& u^{\prime \prime}(\xi)=\frac{a_{0} \mu^{2} \cos (\mu \xi)\left[2 a_{1}^{2}+a_{1} \sin (\mu \xi) a_{2}-a_{2}^{2}\right]}{\left[a_{2}+a_{1} \sin (\mu \xi)\right]^{3}} .
\end{aligned}
$$

in the second form.

We substitute Eqs. (11)or(12)into the reduced form of the governing equation obtained above in Eq. (4). Then, equating the coefficients of each term having the same power of $\cos ^{m}(\mu \xi)$ or $\sin ^{m}(\mu \xi)$ to zero, an algebraic system of equations including all the parameters used in the 
predicted solution, governing equation and the wave transform is solved for $a_{0}, a_{1}, a_{2}, c$ and $\mu$. Determining $a_{0}, a_{1}, a_{2}, c$ and $\mu$ in terms of other parameters and substitution of them into Eq. (9) and (10), one gets solutions for Eq.(2) in rational sin-cos forms.

\section{Exact Solutions}

In this Section, we apply new extended rational trigonometric methods to solve the coupled Higgs equation.

We seek its traveling wave solution of the form

$$
u(x, t)=U(\xi) e^{i \phi}, \quad v(x, t)=V(\xi), \quad \xi=x+c t, \quad \phi=p x+r t
$$

In Eq. (13), $\phi(x, t)$ is the phase component of the soliton, $r$ is the wave number and $p$ is the velocity of the soliton.

We substitute Eq. (13) into Eq. (1)to obtain nonlinear ODEs

$$
\begin{aligned}
& \left(c^{2}-1\right) U^{\prime \prime}+\left(p^{2}-r^{2}\right) U-2 U V+U^{3}=0 \\
& \left(c^{2}+1\right) V^{\prime \prime}-2\left(U^{\prime}\right)^{2}-2 U U^{\prime \prime}=0
\end{aligned}
$$

where prime denotes differentiation with respect to $\xi$.

Integrating the second equation in (16) twice and neglecting the constant of integration, we get

$$
\left(c^{2}+1\right) V=U^{2} \text {. }
$$

Substituting $V$ into the first equation of the system and integrating we obtain

$$
\left(c^{4}-1\right) U^{\prime \prime}+\left(c^{2}+1\right)\left(p^{2}-r^{2}\right) U+\left(c^{2}-1\right) U^{3}=0
$$

Substituting (5) along with (7) into Eq.(16) and collecting all the terms with the same power of $\cosh ^{m}(\mu \xi)$ together, equating each coefficient to zero, yields a set of simultaneous algebraic equations for $c, \mu, r, p$, and $a_{i}, i=0,1,2$, as follows

$$
\begin{aligned}
\text { const: } & c^{2} p^{2} a_{2}^{2}-2 \mu^{2} c^{4} a_{1}^{2}+a_{0}^{2}-c^{2} r^{2} a_{2}^{2}+2 \mu^{2} a_{1}^{2}-r^{2} a_{2}^{2}+p^{2} a_{2}^{2}-\mu^{2} a_{2}^{2} \\
& +\mu^{2} c^{4} a_{2}^{2}-a_{0}^{2} c^{2}=0, \\
\text { cosh : } & -\mu^{2} c^{4} a_{2} a_{1}-2 r^{2} a_{2} a_{1}+2 p^{2} a_{2} a_{1}+\mu^{2} a_{2} a_{1}+2 c^{2} p^{2} a_{2} a_{1}-2 c^{2} r^{2} a_{2} a_{1}=0, \\
\cosh ^{2}: & -a_{0}^{2}-r^{2} a_{1}^{2}-c^{2} r^{2} a_{1}^{2}+c^{2} p^{2} a_{1}^{2}+a_{0}^{2} c^{2}+p^{2} a_{1}^{2}=0 .
\end{aligned}
$$

Solution of the algebraic system given above leads 


\section{Case 1:}

$$
\mu=\sqrt{\frac{2\left(p^{2}-r^{2}\right)}{c^{2}-1}}, \quad a_{0}= \pm \sqrt{-\frac{\left(p^{2}-r^{2}\right)\left(c^{2}+1\right)}{c^{2}-1}} a_{2}, \quad a_{1}= \pm a_{2} .
$$

\section{Case 2:}

$$
\mu=\sqrt{\frac{2\left(p^{2}-r^{2}\right)}{c^{2}-1}}, \quad a_{0}=\mp \sqrt{-\frac{\left(p^{2}-r^{2}\right)\left(c^{2}+1\right)}{c^{2}-1}} a_{2}, \quad a_{1}= \pm a_{2} .
$$

Case 3:

$$
\mu=\frac{\sqrt{2}}{2} \sqrt{\frac{p^{2}-r^{2}}{c^{2}-1}}, \quad a_{0}= \pm \sqrt{-\frac{\left(p^{2}-r^{2}\right)\left(c^{2}+1\right)}{c^{2}-1}} a_{1}, \quad a_{2}=0 .
$$

Combining Eqs. in (17), (13) and (5), the system (1) has the following exact solution

$$
\begin{aligned}
& u(x, t)= \pm \sqrt{-\frac{\left(p^{2}-r^{2}\right)\left(c^{2}+1\right)}{c^{2}-1}} \frac{\sinh \left(\sqrt{\left.\frac{2\left(p^{2}-r^{2}\right)}{c^{2}-1}(x+c t)\right)}\right.}{\left( \pm 1+\cosh \left(\sqrt{\frac{2\left(p^{2}-r^{2}\right)}{c^{2}-1}}(x+c t)\right)\right.} e^{i(p x+r t)}, \\
& v(x, t)=\frac{\left(r^{2}-p^{2}\right)}{c^{2}-1}\left(\frac{\sinh \left(\sqrt{\left.\frac{2\left(p^{2}-r^{2}\right)}{c^{2}-1}(x+c t)\right)}\right)^{2}}{\left( \pm 1+\cosh \left(\sqrt{\frac{2\left(p^{2}-r^{2}\right)}{c^{2}-1}(x+c t)}\right)\right)}\right)^{2},
\end{aligned}
$$

Combining Eqs. in (19), (13) and (5), the system (1) has the following exact solution

$$
\begin{aligned}
& u(x, t)= \pm \sqrt{-\frac{\left(p^{2}-r^{2}\right)\left(c^{2}+1\right)}{c^{2}-1}} \tanh \left(\frac{\sqrt{2}}{2} \sqrt{\frac{p^{2}-r^{2}}{c^{2}-1}}(x+c t)\right)^{i(p x+r t)}, \\
& v(x, t)=\frac{\left(r^{2}-p^{2}\right)}{c^{2}-1} \tanh ^{2}\left(\frac{\sqrt{2}}{2} \sqrt{\frac{p^{2}-r^{2}}{c^{2}-1}}(x+c t)\right),
\end{aligned}
$$


Substituting (6) along with (8) into Eq.(16) and collecting all the terms with the same power of $\cosh ^{m}(\mu \xi)$ or $\sinh ^{m}(\mu \xi)$ together, equating each coefficient to zero, yields a set of simultaneous algebraic equations for $c, \mu, r, p$, and $a_{i}, i=0,1,2$, as follows

$$
\begin{aligned}
& \text { const: } c^{2} r^{2} a_{2}^{2}-c^{2} p^{2} a_{2}^{2}-\mu^{2} c^{4} a_{2}^{2}-2 \mu^{2} c^{4} a_{1}^{2}+p^{2} a_{1}^{2}-r^{2} a_{1}^{2}-p^{2} a_{2}^{2}+r^{2} a_{2}^{2}+\mu^{2} a_{2}^{2} \\
& +2 \mu^{2} a_{1}^{2}+c^{2} p^{2} a_{1}^{2}-c^{2} r^{2} a_{1}^{2}=0, \\
& \text { sinh : }-2 c^{2} p^{2} a_{2} a_{1}+2 c^{2} r^{2} a_{2} a_{1}-2 p^{2} a_{2} a_{1}+2 r^{2} a_{2} a_{1}+\mu^{2} c^{4} a_{2} a_{1}-\mu^{2} a_{2} a_{1}=0 \text {, } \\
& \cosh ^{2}:-c^{2} p^{2} a_{1}^{2}+c^{2} r^{2} a_{1}^{2}-p^{2} a_{1}^{2}+r^{2} a_{1}^{2}-a_{0}^{2} c^{2}+a_{0}^{2}=0 \text {. }
\end{aligned}
$$

Solving the system of algebraic equations gives

\section{Case 1:}

$$
\mu=\sqrt{\frac{2\left(p^{2}-r^{2}\right)}{c^{2}-1}}, \quad a_{0}= \pm \sqrt{-\frac{\left(p^{2}-r^{2}\right)\left(c^{2}+1\right)}{c^{2}-1}} a_{2}, \quad a_{1}= \pm i a_{2} .
$$

\section{Case 2:}

$$
\mu=\sqrt{\frac{2\left(p^{2}-r^{2}\right)}{c^{2}-1}}, \quad a_{0}=\mp \sqrt{-\frac{\left(p^{2}-r^{2}\right)\left(c^{2}+1\right)}{c^{2}-1}} a_{2}, \quad a_{1}= \pm i a_{2} .
$$

Case 3:

$$
\mu=\frac{\sqrt{2}}{2} \sqrt{\frac{p^{2}-r^{2}}{c^{2}-1}}, \quad a_{0}= \pm \sqrt{-\frac{\left(p^{2}-r^{2}\right)\left(c^{2}+1\right)}{c^{2}-1}} a_{1}, \quad a_{2}=0 .
$$

Combining Eqs. in (24), (13) and (6), the system (1) has the following exact solution

$$
u(x, t)= \pm \sqrt{-\frac{\left(p^{2}-r^{2}\right)\left(c^{2}+1\right)}{c^{2}-1}} \frac{\cosh \left(\sqrt{\left.\frac{2\left(p^{2}-r^{2}\right)}{c^{2}-1}(x+c t)\right)}\right.}{\left( \pm 1+i \sinh \left(\sqrt{\frac{2\left(p^{2}-r^{2}\right)}{c^{2}-1}}(x+c t)\right)\right.} e^{i(p x+r t)},
$$




$$
v(x, t)=\frac{\left(r^{2}-p^{2}\right)}{c^{2}-1}\left(\frac{\cosh \left(\sqrt{\left.\frac{2\left(p^{2}-r^{2}\right)}{c^{2}-1}(x+c t)\right)}\right.}{\left( \pm 1+i \sinh \left(\sqrt{\frac{2\left(p^{2}-r^{2}\right)}{c^{2}-1}}(x+c t)\right)\right.}\right)^{2},
$$

Combining Eqs. in (26), (13) and (6), the system (1) has the following exact solution

$$
\begin{aligned}
& u(x, t)= \pm \sqrt{-\frac{\left(p^{2}-r^{2}\right)\left(c^{2}+1\right)}{c^{2}-1}} \operatorname{coth}\left(\frac{\sqrt{2}}{2} \sqrt{\frac{p^{2}-r^{2}}{c^{2}-1}}(x+c t)\right) e^{i(p x+r t),} \\
& v(x, t)=\frac{\left(r^{2}-p^{2}\right)}{c^{2}-1} \operatorname{coth}^{2}\left(\frac{\sqrt{2}}{2} \sqrt{\frac{p^{2}-r^{2}}{c^{2}-1}}(x+c t)\right),
\end{aligned}
$$

Substituting (9) along with (11) into Eq.(16) and collecting all the terms with the same power of $\cos ^{m}(\mu \xi)$ together, equating each coefficient to zero, yields a set of simultaneous algebraic equations for $c, \mu, r, p$, and $a_{i}, i=0,1,2$, as follows

$$
\begin{aligned}
& \text { const : } c^{2} p^{2} a_{2}{ }^{2}-c^{2} r^{2} a_{2}^{2}+p^{2} a_{2}{ }^{2}-r^{2} a_{2}^{2}-a_{0}{ }^{2}-\mu^{2} c^{4} a_{2}^{2}+\mu^{2} a_{2}{ }^{2}-2 a_{1}^{2} \mu^{2}+a_{0}{ }^{2} c^{2} \\
& +2 a_{1}^{2} \mu^{2} c^{4}=0 \text {, } \\
& \cos :-2 a_{1} c^{2} r^{2} a_{2}+2 a_{1} p^{2} a_{2}+2 a_{1} c^{2} p^{2} a_{2}-a_{1} \mu^{2} a_{2}-2 a_{1} r^{2} a_{2}+a_{1} \mu^{2} c^{4} a_{2}=0, \\
& \cos ^{2}: a_{1}^{2} c^{2} p^{2}-a_{1}^{2} c^{2} r^{2}+a_{1}^{2} p^{2}-a_{1}^{2} r^{2}-a_{0}^{2} c^{2}+a_{0}^{2}=0 \text {. }
\end{aligned}
$$

Solution of the algebraic system given above leads

\section{Case 1:}

$$
\mu=\sqrt{-\frac{2\left(p^{2}-r^{2}\right)}{c^{2}-1}}, \quad a_{0}= \pm \sqrt{\frac{\left(p^{2}-r^{2}\right)\left(c^{2}+1\right)}{c^{2}-1}} a_{2}, \quad a_{1}= \pm a_{2} .
$$

\section{Case 2:}

$$
\mu=\sqrt{-\frac{2\left(p^{2}-r^{2}\right)}{c^{2}-1}}, \quad a_{0}=\mp \sqrt{\frac{\left(p^{2}-r^{2}\right)\left(c^{2}+1\right)}{c^{2}-1}} a_{2}, \quad a_{1}= \pm a_{2} .
$$

\section{Case 3:}




$$
\mu=\frac{\sqrt{2}}{2} \sqrt{-\frac{p^{2}-r^{2}}{c^{2}-1}}, \quad a_{0}= \pm \sqrt{\frac{\left(p^{2}-r^{2}\right)\left(c^{2}+1\right)}{c^{2}-1}} a_{1}, \quad a_{2}=0 .
$$

Combining Eqs. in (31), (13) and (9), the system (1) has the following exact solution

$$
\begin{aligned}
& u(x, t)= \pm \sqrt{\frac{\left(p^{2}-r^{2}\right)\left(c^{2}+1\right)}{c^{2}-1}} \frac{\sin \left(\sqrt { - \frac { 2 ( p ^ { 2 } - r ^ { 2 } ) } { c ^ { 2 } - 1 } ( x + c t ) ) } \left( \pm 1+\cos \left(\sqrt{-\frac{2\left(p^{2}-r^{2}\right)}{c^{2}-1}}(x+c t)\right)\right.\right.}{\left(c^{2}\right)} e^{i(p x+r t)}, \\
& \left.v(x, t)=\frac{\left(p^{2}-r^{2}\right)}{c^{2}-1}\left(\frac{\sin \left(\sqrt{-\frac{2\left(p^{2}-r^{2}\right)}{c^{2}-1}(x+c t)}\right)}{\left( \pm 1+\cos \left(\sqrt{-\frac{2\left(p^{2}-r^{2}\right)}{c^{2}-1}(x+c t)}\right)\right.}\right)^{2}\right)
\end{aligned}
$$

Combining Eqs. in (33), (13) and (9), the system (1) has the following exact solution

$$
\begin{aligned}
& u(x, t)= \pm \sqrt{\frac{\left(p^{2}-r^{2}\right)\left(c^{2}+1\right)}{c^{2}-1}} \tan \left(\frac{\sqrt{2}}{2} \sqrt{-\frac{p^{2}-r^{2}}{c^{2}-1}}(x+c t)\right) e^{i(p x+r t),} \\
& v(x, t)=\frac{\left(p^{2}-r^{2}\right)}{c^{2}-1} \tan ^{2}\left(\frac{\sqrt{2}}{2} \sqrt{-\frac{p^{2}-r^{2}}{c^{2}-1}}(x+c t)\right),
\end{aligned}
$$

Substituting (10) along with (12) into (16) and collecting all the terms with the same power of $\cos ^{m}(\mu \xi)$ or $\sin ^{m}(\mu \xi)$ together, equating each coefficient to zero yields a set of algebraic equations. Solving these equations yields

\section{Case 1:}

$$
\mu=\sqrt{-\frac{2\left(p^{2}-r^{2}\right)}{c^{2}-1}}, \quad a_{0}= \pm \sqrt{\frac{\left(p^{2}-r^{2}\right)\left(c^{2}+1\right)}{c^{2}-1}} a_{2}, \quad a_{1}= \pm a_{2} .
$$

\section{Case 2:}

$$
\mu=\sqrt{-\frac{2\left(p^{2}-r^{2}\right)}{c^{2}-1}}, \quad a_{0}=\mp \sqrt{\frac{\left(p^{2}-r^{2}\right)\left(c^{2}+1\right)}{c^{2}-1}} a_{2}, \quad a_{1}= \pm a_{2} .
$$




\section{Case 3:}

$$
\mu=\frac{\sqrt{2}}{2} \sqrt{-\frac{p^{2}-r^{2}}{c^{2}-1}}, \quad a_{0}= \pm \sqrt{\frac{\left(p^{2}-r^{2}\right)\left(c^{2}+1\right)}{c^{2}-1}} a_{1}, \quad a_{2}=0 .
$$

Combining Eqs. in (38), (13) and (10), the system (1) has the following exact solution

$$
\begin{aligned}
& u(x, t)= \pm \sqrt{\frac{\left(p^{2}-r^{2}\right)\left(c^{2}+1\right)}{c^{2}-1}} \frac{\cos \left(\sqrt{\left.-\frac{2\left(p^{2}-r^{2}\right)}{c^{2}-1}(x+c t)\right)}\right.}{\left( \pm 1+\sin \left(\sqrt{-\frac{2\left(p^{2}-r^{2}\right)}{c^{2}-1}}(x+c t)\right)\right.} e^{i(p x+r t)}, \\
& v(x, t)=\frac{\left(p^{2}-r^{2}\right)}{c^{2}-1}\left(\frac{\cos \left(\sqrt{-\frac{2\left(p^{2}-r^{2}\right)}{c^{2}-1}(x+c t)}\right)}{\left( \pm 1+\sin \left(\sqrt{-\frac{2\left(p^{2}-r^{2}\right)}{c^{2}-1}(x+c t)}\right)\right)}\right)^{2},
\end{aligned}
$$

NowCombining Eqs. in (40), (13) and (10), the system (1) has the following exact solution

$$
\begin{aligned}
& u(x, t)= \pm \sqrt{\frac{\left(p^{2}-r^{2}\right)\left(c^{2}+1\right)}{c^{2}-1}} \cot \left(\frac{\sqrt{2}}{2} \sqrt{-\frac{p^{2}-r^{2}}{c^{2}-1}}(x+c t)\right) e^{i(p x+r t)}, \\
& v(x, t)=\frac{\left(p^{2}-r^{2}\right)}{c^{2}-1} \cot ^{2}\left(\frac{\sqrt{2}}{2} \sqrt{-\frac{p^{2}-r^{2}}{c^{2}-1}}(x+c t)\right),
\end{aligned}
$$

\section{Physical comments on graphical illustrations}

We illustrate some particular forms of the solutions in order to comprehend better. The solutions are plotted in three dimensional space for some particular selections of parameters.

\section{a. Solution given in equations 20-21}

We plot real and imaginary componentsseparately, Fig 1-2, and modulus of $u(x, t)$, Fig 3 , and $v(x, t)$, Fig4, for the parameters $p=0.1, r=0.2, c=2, v=2$ in the finite space $x \in[-20,20] \times t \epsilon[0,5]$. The solution $u(x, t)$ is in the complex form whose real and imaginary components represent multi wave solutions. As a result, the modulus is also in form of multi waves. The solution $v(x, t)$ is in the real form and represent single solitary wave of negative amplitude moving along the space axis with a constant velocity $c$, Fig 4 . 


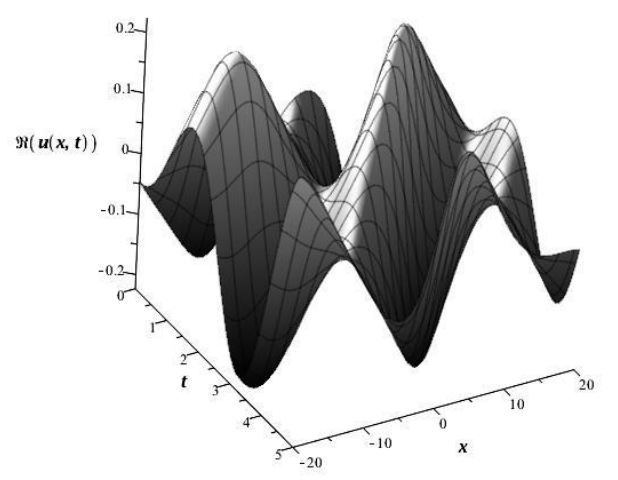

Fig 1. Real component of $u(x, t)$

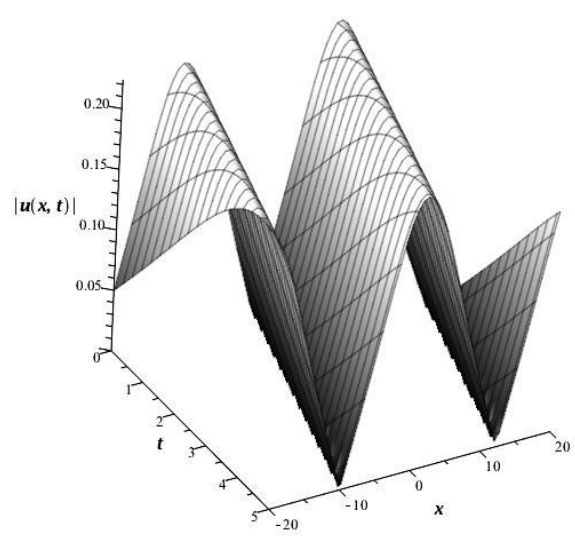

Fig 3. Modulus of $u(x, t)$

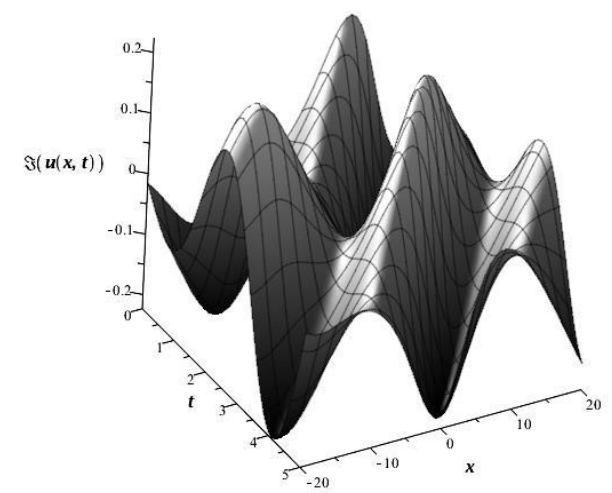

Fig 2. Imaginary component of $u(x, t)$

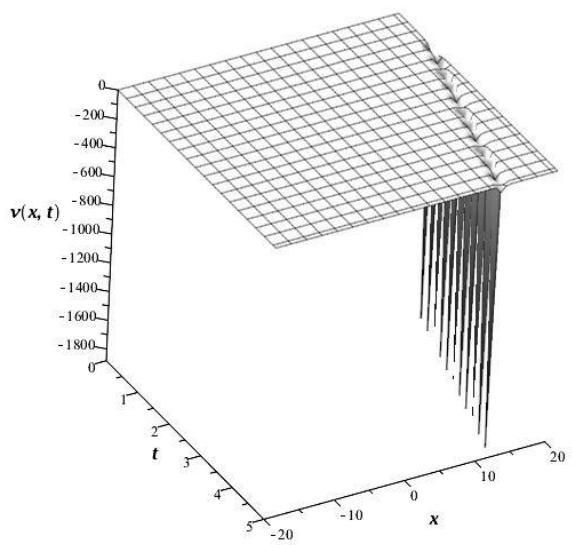

Fig 4. $v(x, t)$

\section{b. Solution given in equations 22-23}

The solution given in equation (22) is plotted for some particular choices of parameters as $p=$ $1 / 10, r=1 / 4, c=1 / 5, v=1$ in the finite domain $x \in[0,40] \times t \epsilon[0,25]$. The Figures 5-8 demonstrate the motion of multi waves of $u(x, t)$ as Figure 8 indicates the motion of $v(x, t)$ in the same domain with the same parameters.
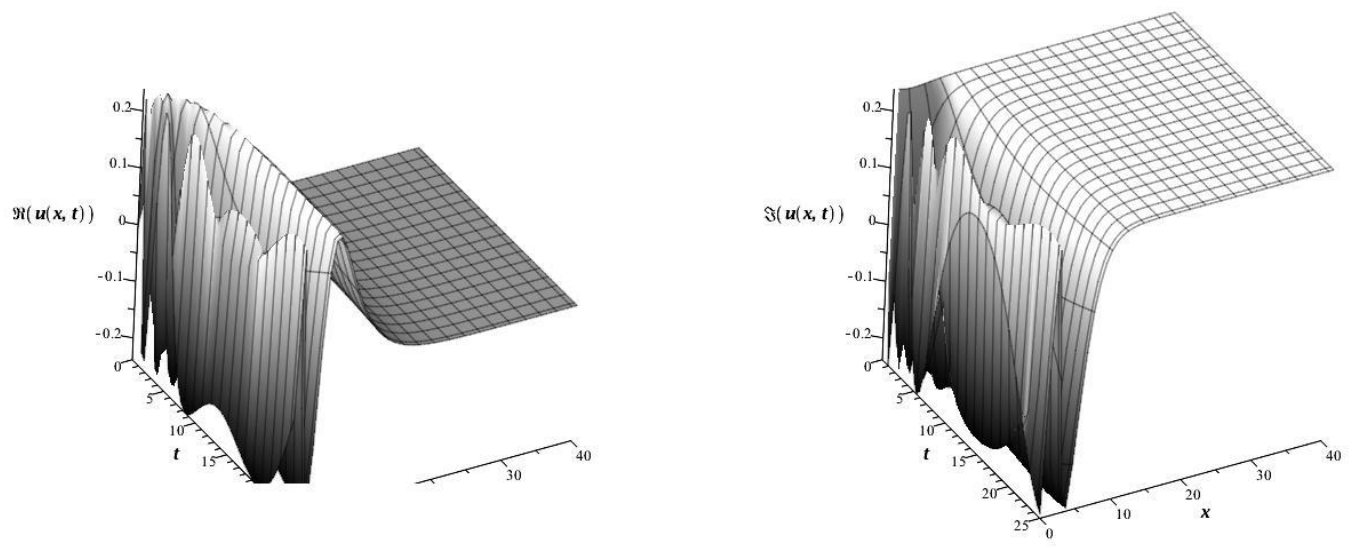
Fig 5. Real component of $u(x, t)$

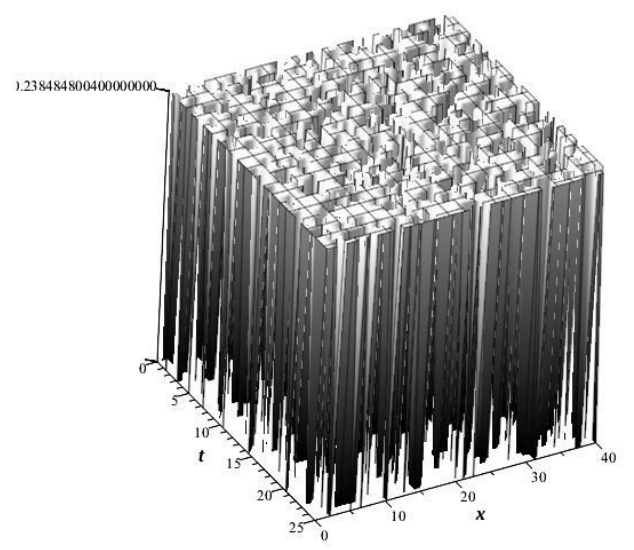

Fig 7. Modulus of $u(x, t)$
Fig 6. Imaginary component of $u(x, t)$

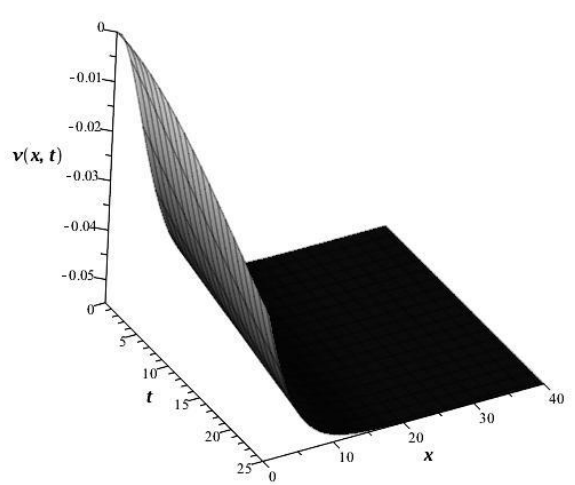

Fig 8. $v(x, t)$

\section{c. Solution given in equations $22-23$}

The solution given in equation (34-35) is plotted for some particular choices of parameters as $p=$ $1 / 10, r=1 / 2, c=1 / 4, v=1$ in the finite domain $x \in[0,50] \times t \in[0,10]$. The Figures $9-11$ demonstrate the motion of multi waves of $u(x, t)$ as Figure 12 indicates the motion of $v(x, t)$ in the same domain with the same parameters. Both real and imaginary components of $u(x, t)$ are of the form of sinusoidal curve moving along the space axis as time proceeds. The modulus of $u(x, t)$ is also depicted in Fig 11. Fig 12 is the simulation of $v(x, t)$ with the same selection of parameters in the same finite domain.

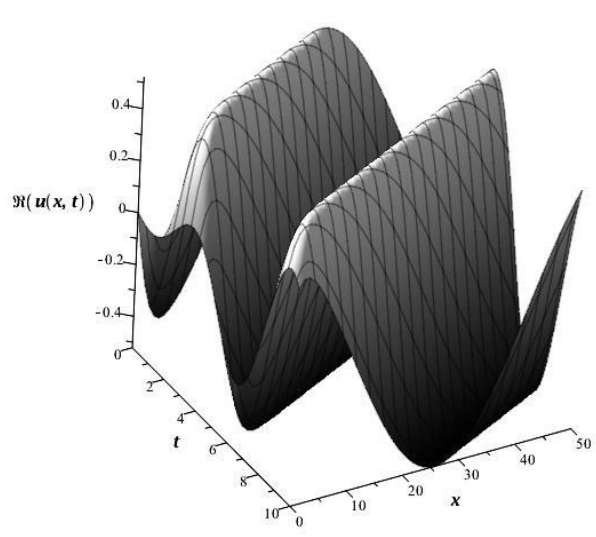

Fig 9. Real component of $u(x, t)$

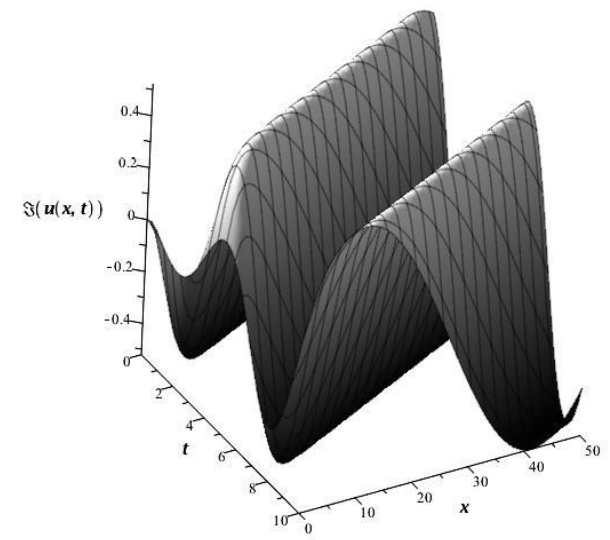

Fig 10 . Imaginary component of $u(x, t)$ 


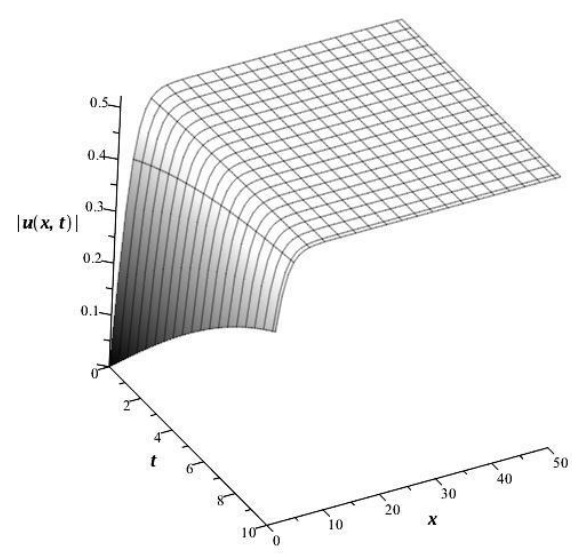

Fig 11. Modulus of $u(x, t)$

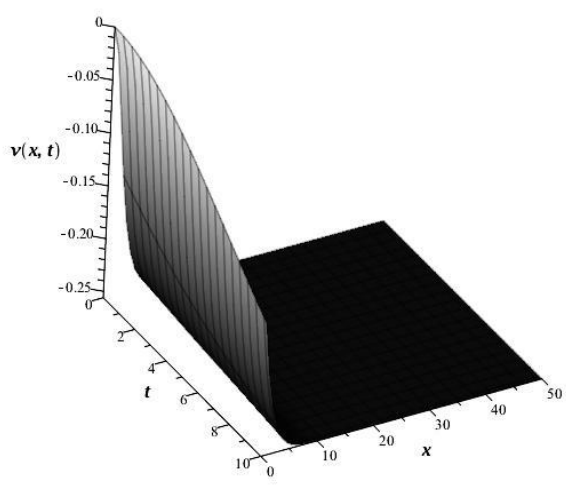

Fig 12. $v(x, t)$

\section{Conclusions}

The new extended rational methods with trigonometric or hyperbolic functions are used to construct the solutions. The logic of the method is simple. The predicted solutions of the forms of rational forms of trigonometric or hyperbolic functions with various parameters are substituted into the governing ODEs derived from the governing system by compatible traveling wave transforms. The remaining steps of the procedure is to determine the unknown parameters and the relations among them by using algebraic methods.

Eventually, in this article, we derive some solutions to the coupled Higgs system in rational forms of trigonometric and hyperbolic functions solutions. Some of the solutions are of the forms in complex valued functions whose components represent solitary or multi waves as some are only real valued solutions. The plots of some particular solutions are used to comprehend the motion.

\section{Acknowledgments}

The authors would like to thank the anonymous reviewers for their useful comments. This research work has been supported by a research grant from the University of Mazandaran.

\section{References}

[1] Tajiri, M. (1983). On N-soliton solutions of coupled Higgs field equation. Journal of the Physical Society of Japan, 52(7), 2277-2280.

[2] Xu, G. Q. (2014). New types of doubly periodic standing wave solutions for the coupled Higgs field equation. In Abstract and Applied Analysis, 2014(769561), 1-8.

[3] Mu, G., \& Qin, Z. (2012). Rogue waves for the coupled Schrödinger-Boussinesq equation and the coupled Higgs equation. Journal of the Physical Society of Japan, 81(8), 084001.

[4] Kumar, S., Singh, K., \& Gupta, R. K. (2012). Coupled Higgs field equation and Hamiltonian amplitude equation: Lie classical approach and $\left(\mathrm{G}^{\prime} / \mathrm{G}\right)$-expansion method. Pramana, 79(1), 41-60. 
[5] Tang, S., \& Xia, S. (2011). Bifurcations of Traveling Wave Solutions for the Coupled Higgs Field Equation. International Journal of Differential Equations, 2011.

[6] Khajeh, A., Kabir, M. M., \&Koma, A. Y. (2010). New exact and explicit travelling wave solutions for the coupled Higgs equation and a nonlinear variant of the PHI-four equation. International Journal of Nonlinear Sciences and Numerical Simulation, 11(9), 725-742.

[7] Hon, Y. C., \& Fan, E. G. (2009). A series of exact solutions for coupled Higgs field equation and coupled Schrödinger-Boussinesq equation. Nonlinear Analysis: Theory, Methods \& Applications, 71(7-8), 3501-3508.

[8] Hu, X. B., Guo, B. L., \& Tam, H. W. (2003). Homoclinic orbits for the coupled SchrödingerBoussinesq equation and coupled Higgs equation. Journal of the Physical Society of Japan, 72(1), 189-190.

[9] Jabbari, A., Kheiri, H., \&Bekir, A. (2011). Exact solutions of the coupled Higgs equation and the Maccari system using He's semi-inverse method and $\left(\mathrm{G}^{\prime} / \mathrm{G}\right)$-expansion method. Computers \& Mathematics with Applications, 62(5), 2177-2186.

[10] Hafez, M. G., Alam, M. N., \& Akbar, M. A. (2015). Traveling wave solutions for some important coupled nonlinear physical models via the coupled Higgs equation and the Maccari system. Journal of King Saud University-Science, 27(2), 105-112.

[11] Aminikhah, H., RefahiSheikhani, A., \&Rezazadeh, H. A. D. I. (2014). Exact solutions of some nonlinear systems of partial differential equations by using the functional variable method. Mathematica, 56, 103-116.

[12] Aminikhah, H., Sheikhani, A. H. R., \&Rezazadeh, H. (2015). Travelling wave solutions of nonlinear systems of PDEs by using the functional variable method. Boletim da SociedadeParanaense de Matemática, 34(2), 213-229.

[13] Eslami, M., Khodadad, F. S., Nazari, F., \&Rezazadeh, H. (2017). The first integral method applied to the Bogoyavlenskii equations by means of conformable fractional derivative. Optical and Quantum Electronics, 49(12), 391.

[14] Rezazadeh, H., Manafian, J., Khodadad, F. S., \&Nazari, F. (2018). Traveling wave solutions for density-dependent conformable fractional diffusion-reaction equation by the first integral method and the improved $\tan (\phi \quad(\xi) / 2)$-expansion method. Optical and Quantum Electronics, 50(3), 121.

[15] Korkmaz, A. (2017). Exact solutions to $(3+1)$ conformable time fractional Jimbo-Miwa, Zakharov-Kuznetsov and modified Zakharov-Kuznetsov equations. Communications in Theoretical Physics, 67(5), 479. 
[16] Korkmaz, A. (2018). On the wave solutions of conformable fractional evolution equations. Communications, 67, 68-79.

[17] Mirzazadeh, M., Eslami, M., \& Biswas, A. (2015). 1-Soliton solution of KdV6 equation. Nonlinear Dynamics, 80(1-2), 387-396.

[18] Korkmaz, A. (2018). Complex Wave Solutions to Mathematical Biology Models I: NewellWhitehead-Segel and Zeldovich Equations. Journal of Computational and Nonlinear Dynamics, 13(8), 081004.

[19] Aminikhah, H., Sheikhani, A. R., \&Rezazadeh, H. (2016). Sub-equation method for the fractional regularized long-wave equations with conformable fractional derivatives. ScientiaIranica. Transaction B, Mechanical Engineering, 23(3), 1048.

[20] Rezazadeh, H., Korkmaz, A., Eslami, M., Vahidi, J., \&Asghari, R. (2018). Traveling wave solution of conformable fractional generalized reaction Duffing model by generalized projective Riccati equation method. Optical and Quantum Electronics, 50(3), 150.

[21] Korkmaz, A., \& Hosseini, K. (2017). Exact solutions of a nonlinear conformable timefractional parabolic equation with exponential nonlinearity using reliable methods. Optical and Quantum Electronics, 49(8), 278.

[22] Guner, O., Korkmaz, A., \&Bekir, A. (2017). Dark soliton solutions of space-time fractional Sharma-Tasso-Olver and potential Kadomtsev-Petviashvili equations. Communications in Theoretical Physics, 67(2), 182.

[23] Guner, O., Bekir, A., \&Korkmaz, A. (2017). Tanh-type and sech-type solitons for some spacetime fractional PDE models. The European Physical Journal Plus, 132(2), 92.

[24] Osman, M. S., Korkmaz, A., Rezazadeh, H., Mirzazadeh, M., Eslami, M., \& Zhou, Q. (2018). The Unified Method for Conformable Time Fractional Schrödinger Equation with Perturbation Terms. Chinese Journal of Physics, 56(5), 2500.

[25] Darvishi, M. T., Najafi, M., \&Wazwaz, A. M. (2018). New extended rational trigonometric methods and applications. Waves in Random and Complex Media, 1-22. 